\title{
MEMS CURRICULUM AT AUBURN UNIVERSITY'S MICROELECTRONICS CENTER
}

\author{
Ramesh Ramadoss \\ Alabama Microelectronics Science and Technology Center \\ Department of Electrical and Computer Engineering \\ Auburn University \\ 200 Broun Hall, Auburn, AL 36849, USA \\ email: ramadra@auburn.edu, Tel: 334-844-1879, Fax: 334-844-1809
}

\begin{abstract}
In this paper, a MEMS curriculum developed at the Alabama Microelectronics Science and Technology Center, Department of Electrical and Computer Engineering, Auburn University is discussed. The course curriculum consists of three major components - class lectures (2 lectures per week), Computer-aided design (CAD) sessions and laboratory sessions. CAD sessions and laboratory sessions are conducted on alternate weeks. The objective of this curriculum is to provide a comprehensive understanding of various aspects of MEMS such as principle of operation, design, modeling, simulation, fabrication and characterization. In class lectures, concepts underlying the principle of operation and design of various MEMS devices are discussed. In CAD sessions, industry standard MEMS software is used for design and simulation of MEMS devices. In laboratory sessions, students are trained to carry out hands-on fabrication and characterization of MEMS devices. These three components are systematically structured to enable integrative learning of various materials presented in this course.
\end{abstract}

\section{INTRODUCTION}

Micro-Electro-Mechanical Systems (MEMS) is a rapidly emerging field that utilizes micromachining techniques to fabricate microsensors and microactuators. Micromachining and mechanical movement of microstructures are the key features of MEMS technology. Typical sizes for MEMS devices range from micrometers to millimeters. The advantages of MEMS devices are low cost, high performance, small size, and low weight compared to conventional devices. MEMS has proved to be a revolutionary technology in many application arenas, including accelerometers, pressure sensors, micro-optics, inkjet nozzles, optical scanners, and fluid pumps. MEMS has made a significant impact in many fields, including telecommunications, optics, robotics, and medicine.

In recent years there has been a considerable interest in the development of MEMS curriculum for training next generation engineers needed for the rapidly growing MEMS industry. MEMS curriculum developed at various universities have been discussed in [1]-[3]. In this paper, the MEMS curriculum developed at the Alabama Microelectronics Science and Technology Center, Department of Electrical and Computer Engineering, Auburn University is discussed. In the following sections, course structure and future course development efforts are discussed in detail.

\section{COURSE STRUCTURE}

The curriculum consists of three major components - class lectures (2 lectures per week), CAD sessions and laboratory sessions. CAD sessions and laboratory sessions are conducted for three hours on alternate weeks. This 4.0 credit hour course typically spans 16 weeks (one semester).

\section{Lecture Component}

In class lectures, basic concepts underlying the principle of operation and design of various MEMS devices are discussed. The topics covered in the class lectures are shown in Table 1. A majority of the topics are covered from the suggested textbook [4] and additional topics are also discussed from advanced books [5] and [6]. As shown in the table, the course is divided into three parts, namely, Part I: Microfabrication and Microstructures, Part II: MEMS Actuators, and Part III: MEMS Sensors.

Table 1: Topics discussed in class lectures

Part I: Microfabrication and Microstructures (6 Weeks)

- Introduction to MEMS

- MEMS Fabrication Processes

- Mechanics of Microstructures

- Mechanics of Beams and Membranes

- Vibration of Microstructures

Part II: MEMS Actuators (5 Weeks)

- Electrostatic Actuator

- Electrothermal Actuator

- Piezoelectric Actuator

- Magnetic Actuator

- RF MEMS Resonators and Filters

- Optical MEMS

Part III: MEMS Sensors (5 Weeks)

- $\quad$ MEMS Accelerometers

- MEMS Gyroscopes

- MEMS Pressure Sensors

- $\quad$ MEMS Microphones

In Part I of the course, fundamentals of microelectronic processes, MEMS fabrication processes such as bulk micromachining, surface micromachining and LIGA techniques, and commercial foundry processes are discussed. Basic mechanics of beams and membranes including concepts such as spring constant, effects of residual stresses on cantilever and fixed-fixed beams, and buckling of beams are discussed. Vibration of microstructures and lumped- 
element modeling of second-order systems using spring, mass, and damper are also discussed.

In Part II of the course, various MEMS actuators such as electrostatic, electrothermal, piezoelectric and electromagnetic are discussed. The principle of operation and governing design equations are discussed for various actuators. Then, the use of these actuators in various applications such as RF MEMS and Optical MEMS are also discussed.

In Part III of the course, various MEMS sensors such as accelerometers, gyroscopes, pressure sensors, microphones etc. are discussed. Case study examples such as Freescale Semiconductors pressure sensors and Analog Devices accelerometers and gyroscopes are also discussed.

\section{Computer-aided Design Sessions}

The introduction of computer-aided design tools in the mid 1990s drastically changed the design procedures followed for MEMS. Computer-aided analysis and optimization have replaced the design process of iterative experimental modifications of the initial design. In addition to their impact on design practice in industry, $\mathrm{CAD}$ tools can play a major role in undergraduate and graduate education. The major impact of CAD tools in MEMS education is in making realistic design examples and case studies available to students. Some advantages of using CAD tools in MEMS education are outlined here - i) the students can learn some of the very basic concepts by repeated computations making use of CAD simulation tools, ii) CAD tools allow the students to explore how the design performance will be affected if the value of a design parameter were to be altered either intentionally or because of the unavoidable tolerances in the values of the components in the fabrication process, iii) CAD tools equipped with field visualizers can be used to learn qualitatively about the reasons for the device's behavior by looking at the distribution of the field (such as stress, charge distribution, temperature distribution, etc.).

Table 2: Computer Aided Design and Simulation of MEMS using CoventorWare [8] (6 Weeks)

CAD \#1: CoventorWare getting Started

CAD \#2: MEMElectro ("Tutorial 1: Beam Design")

CAD \#3: MEMMech ("Tutorial 1: Beam Design")

CAD\#4: MEMMech ("Tutorial 4: Modal Analysis")

CAD\#5: Co-SolveEM ("Tutorial 3: Beam Simulation Analysis")

CAD \#6: MemETherm ("Tutorial 6: Temperature Analysis")

An important aspect of Auburn University's MEMS curriculum is the use of ConventorWare [7], a very widely used MEMS design software for computer-aided design, analysis and optimization of MEMS devices. CoventorWare consists of multi-physics numerical analysis tools such as MemElectro (electrostatic solver), MemMech (mechanical and thermo-mechanical solver), Co-Solve EM (coupled eletromechanical solver), MemETherm (electrothermo-mechanical solver), etc. CAD assignments involve design, simulation and optimization of various MEMS devices. A list of CAD assignments performed using CoventorWare is shown in Table 2. A majority of the CAD assignments are based on CoventorWare tutorials. The CAD assignments are designed such that the students are exposed to pertinent tools after discussion of the relevant topics in the lectures. For example, after completion of the lectures on basic beam mechanics in Part I the students perform mechanical analysis of a beam using MEMMech in CAD \#2. Similarly, the students perform electro-mechanical analysis of an electrostatic actuator in CAD\#5 after completion of class lectures on electrostatic actuators in Part II.

Top View Cross Section

a) Silicon wafer $<100>$

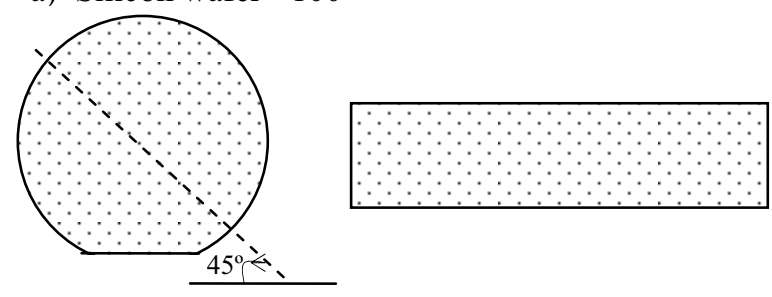

b) Nitride Deposition

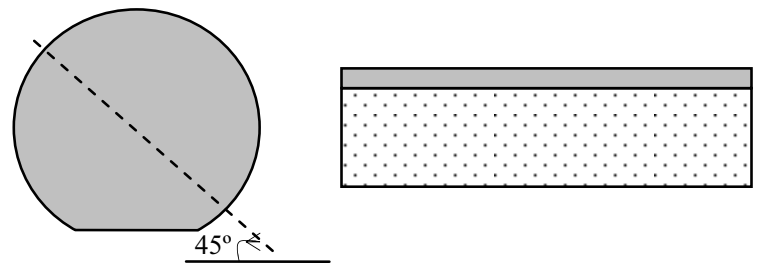

c) Pattern Nitride

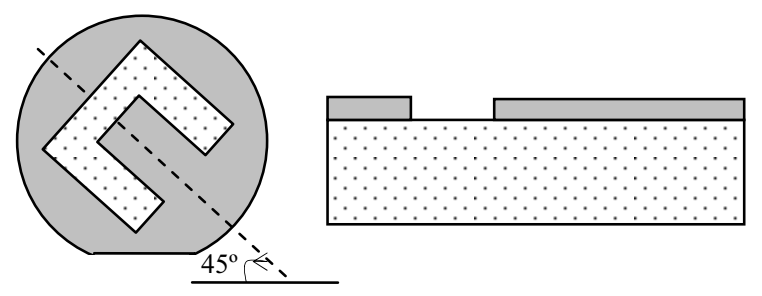

d) Anisotropic Etching

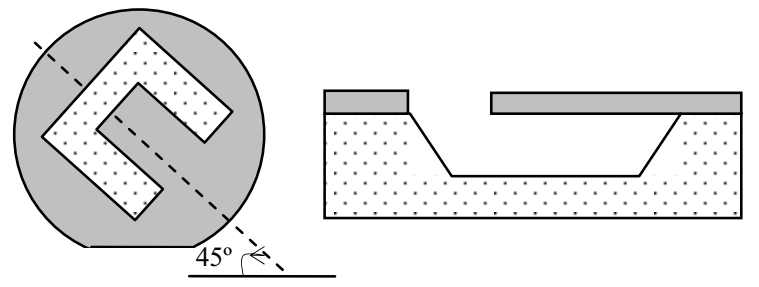

Figure 1: Process flow for bulk micromachining (a) starting substrate $<100>$, (b) silicon nitride deposition using LPCVD, (c) patterning silicon nitride layer by photolithography, (d) anisotropic etching of silicon substrate using $\mathrm{KOH}$.

\section{Laboratory Sessions}

The laboratory sessions provide hands-on experience in the fabrication and testing of MEMS devices using the facilities available at the Alabama Microelectronics Science and Technology Center (AMSTC) [7]. The major pieces of equipment 
used in this course include Karl Suss MA/BA 6 frontside/backside mask aligner, Thermco oxidation and diffusion chamber, Tempress LPCVD system, CHA Industries's Mark 50 dual Ebeam/sputter/ion gun deposition system, and STS AOE 100 DRIE system.

The objective of the laboratory sessions is to provide fabrication experience in two most commonly used MEMS fabrication process, namely, bulk micromachining and surface micromachining. Before the start of lab sessions, all the students are required to read the lab safety rules and guidelines. A quiz was conducted to assess students' understanding of safety rules.

Table 3: Laboratory sessions (9 Weeks)

MEMS Fabrication

LAB \#1: Introduction to MEMS fabrication facilities

LAB \#2-3: Bulk micromachining (2 weeks)

LAB \#4-6: Surface micromachining (3 Weeks)

Characterization and Testing

LAB \#7: Profile Characterization Using WYKO Optical Profiler

LAB \#8: MEMS Actuator Testing

LAB \#9: MEMS Pressure Sensor Characterization

LAB \#10: MEMS TriaxBoard Accelerometer Demonstration
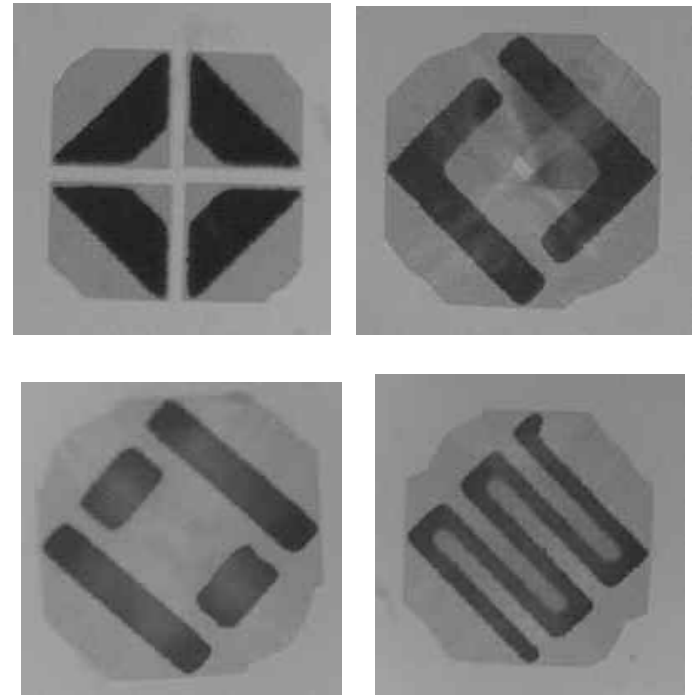

Figure 2: Suspended MEMS structures fabricated using bulk micromachining

In bulk micromachining sessions, the objective is to fabricate suspended microstructures in the silicon substrate. The major process steps involved in this lab are shown in Figure 1. A $<100>$ silicon wafer is used as the starting substrate and silicon nitride is deposited by an LPCVD process. The silicon nitride layer is patterned using standard photolithographic steps such as spincoating, UV exposure using mask aligner, photoresist development, and plasma etching. Finally, the patterned silicon nitride is used as a masking layer and anisotropic etching of silicon substrate is performed using $\mathrm{KOH}$. Bulk micromachining lab consists of two sessions performed in two weeks. In the first session, the students learn basic steps such as wafer cleaning, silicon nitride deposition, and photolithography. In the second session, the students carry out plasma etching of silicon nitride and anisotropic etching of the silicon substrate. Photographs of various suspended microstructures fabricated after the completion of the bulk micromachining sessions are shown in Figure 2.

(a) Silicon Dioxide Growth

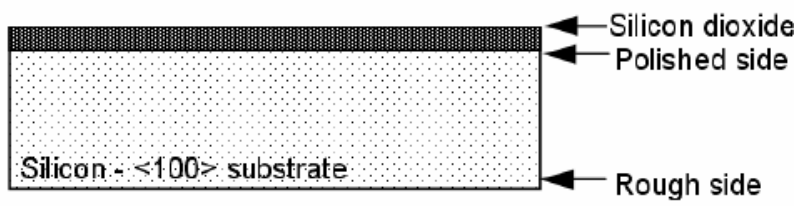

(b) Pattern Photoresist Layer

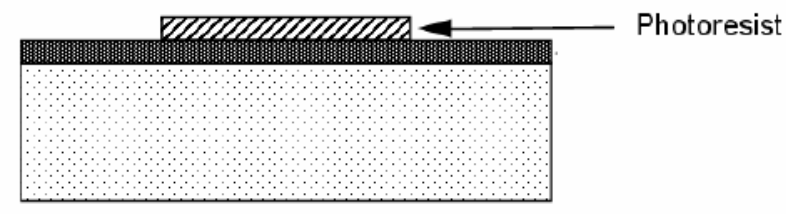

(c) Aluminum Film Deposition

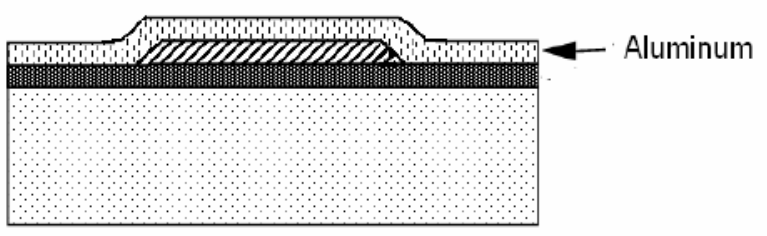

(d) Pattern and Release

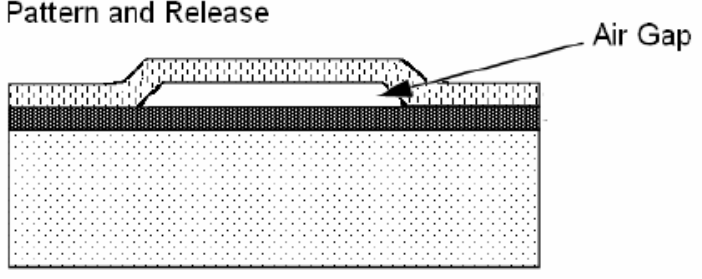

Figure 3: Process flow for surface micromachining (a) silicon dioxide growth using oxidation furnace, (b) defining photoresist sacrificial layer, (c) e-beam deposition of the aluminum structural layer, (d) pattern and release aluminum beams by etching the sacrificial layer.

In surface micromachining sessions, the objective is to fabricate electrostatic MEMS actuators using aluminum as the structural layer. Surface micromachining lab consists of three sessions performed in three weeks. The process flow involved in the fabrication of a clamped-clamped structure by surface micromachining is shown in Figure 3. A thin layer of silicon dioxide is grown on the starting $<100>$ silicon substrate and anchor openings are etched through the oxide layer. The concept of sacrificial layer (soluble/removable) is the basis for fabrication of freestanding microstructures by surface micromachining. A sacrificial photoresist layer is defined on the oxide layer by photolithography. Openings are etched entirely through the sacrificial photoresist layer to provide anchoring point for the structural layer. A thin film of aluminum structural material is deposited and etched to define the required MEMS structure. After etching the photoresist sacrificial layer, the patterned structure is 
separated from the substrate (except at the anchoring point) by the thickness of the removed sacrificial layer to form a freestanding structure. Photographs of the fabricated clamped-clamped and cantilever beams are shown in Figure 4.

After completion of all the fabrication sessions, 'Characterization and Testing' sessions are conducted on profile characterization of microstructures using a WYKO optical profilometer and testing of electrostatic actuators using a probe station. Additional laboratory sessions focusing on the characterization of commercially available MEMS devices such as Freescale semiconductor pressure sensors and accelerometers are also conducted.

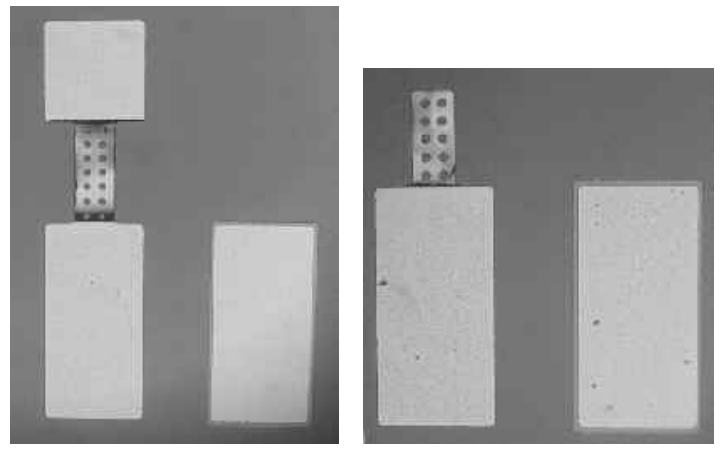

Figure 4: Clamped-clamped beam (left) and cantilever beam (right) fabricated using surface micromachining

A website is being maintained for this course [8]. Typically the materials posted on the website include the course syllabus, weekly schedule, homework assignments, lecture notes, laboratory notes etc. The students have access to and download the needed material at any time and at any place at their convenience.

\section{FUTURE COURSE DEVELOPMENT}

Our department has been offering a "Microelectronics Fabrication" course in spring and fall semesters for several years [10]. The infrastructure developed for this course facilitated the implementation of a new MEMS curriculum with hands-on laboratory components. For the past two years, the MEMS course has been experimentally taught as a special topics course once a year. In spring 2005, a total of 20 students from various disciplines such as electrical engineering, mechanical engineering, chemical engineering, and materials engineering were enrolled in this course. Overall, the students find the CAD sessions and laboratory sessions very useful in learning MEMS. The contents covered in the current course structure could be significantly expanded and offered as two MEMS courses "MEMS I: MEMS sensors and actuators" and "MEMS II: MEMS Fabrication". MEMS I could be designed as entry level course for senior-undergraduate and graduate students from various disciplines. MEMS I could emphasize more on the fundamentals of micromachining and MEMS devices. The second course "MEMS II" could focus on providing hands-on laboratory sessions. In "MEMS II" advanced fabrication process could be designed for fabrication of complex MEMS devices. CAD sessions could be included in both courses. MEMS I could introduce the students to device level design and simulation of MEMS devices and MEM II could focus on system level design and simulation of MEMS based Microsystems.

\section{CONCLUSION}

A MEMS curriculum consisting of three major components - class lectures, Computer-aided design (CAD) sessions and laboratory sessions is discussed. The introduction of CAD sessions and hands-on laboratory sessions in MEMS education would enable us in bridging the gap between classroom instruction of MEMS and the practice of MEMS in industry. MEMS curriculum that meets the needs of industry would facilitate rapid advancement of the state of technology.

\section{ACKNOWLEDGEMENT(S)}

The author would like to thank Charles Ellis, Manager, Alabama Microelectronics Science and Technology center at Auburn University for his help in conducting the laboratory sessions, and the teaching assistants Lia Almeida and Ron Jackson for their help in developing the laboratory sessions. The author would also like to thank Dr. R. C. Jaeger for reviewing this manuscript and Dr. Y. C. Lee, University of Colorado at Boulder, for providing CAD assignments from his MEMS course.

\section{REFERENCES}

[1]. P. J. Hesketh, J. G. Boyd, M. J. McNallan, and G. J. Maclay, "Curriculum development in MEMS," Electrochem. Soc. Interface, vol. 6, no. 1, pp. 48-51, Spring issue 1997.

[2]. C. Liu, "Integration of a MEMS education curriculum with interdisciplinary research," in Proc. 1997 Biennial Univ./Government/Ind. Microelectron. Symp., 1997, pp. 137-140. [3]. Liwei Lin, "Curriculum Development in Microelectromechanical Systems in Mechanical Engineering," IEEE Transactions on Education, Vol. 44, no. 1, Feb. 2001, pp. 61-66.

[4]. Tai-Ran Hsu, "MEMS and Microsystems: Design and Manufacture, McGraw-Hill, 1st edition, ISBN: 0072393912.

[5]. Stephen D. Senturia, "Microsystem Design," Kluwer Academic Publishers, January 2001, ISBN: 0792372468.

[6]. Min-Hang Bao, "Micro Mechanical Transducers: Pressure Sensors, Accelerometers and Gyroscopes," Elsevier Science (Handbook of Sensors and Actuators Series) ISBN: 044450558X, October 2000.

[7]. MEMS fabrication, assembly, and packaging facilities at the Alabama Microelectronics Science and Technology center, Department of Electrical and Computer Engineering, Auburn University: http://spider.eng.auburn.edu/amstc/

[8]. "MEMS Sensors and Actuators" course website: http://www.eng.auburn.edu/ ramadra/mems/

[9]. CoventorWare ver. 2005, Coventor Inc., NC, USA.

[10]. R. C. Jaeger, Y. Tzeng, K. Daneshvar, T. A. Baginski and J. L. Davidson, "The Alabama Microelectronics Science and Technology Center and the microelectronics program at Auburn University," IEEE UGIM '85 Proceedings, pp. 42-45, June 1985. 